\title{
DIFERENCIAS EN EL HÁBITO DE CRECIMIENTO COMO VARIABLE eXPLICATIVA de LA INFLUENCIA CLIMÁtica en PROSOPIS FLEXUOSA EN el Desierto del Monte Central (Argentina)
}

\author{
SERGIO PIRAINO ${ }^{1,2}$ y FIDEL ALEJANDRO ROIG JUÑENT ${ }^{1}$
}

\begin{abstract}
Resumen: Los métodos dendroclimatológicos representan una de las herramientas más valiosas para analizar la influencia del clima sobre la dinámica del incremento radial y la morfología de las plantas leñosas. En el Desierto del Monte Central, Prosopis flexuosa DC (algarrobo dulce), crece como árbol uni-fustal pero también como multi-fustal. Investigaciones anteriores han evidenciado la utilidad del ancho de anillo de esta especie como registro de eventos climáticos. Sin embargo, estas investigaciones han diferido metodológicamente, considerando o descartando los individuos multi-fustales. Este trabajo analizó si la variabilidad en el hábito de crecimiento expresa diferencias en la señal dendroclimática del algarrobo dulce. Dos cronologías de ancho de anillos, una derivada de individuos uni-fustales (UF) y otra de multi-fustales (MF) presentes en un mismo ambiente ecológico fueron desarrolladas. Se observó que hay una mayor sensibilidad de la cronología MF respecto de la UF en relación a la incidencia en el crecimiento de eventos climáticos extremos y del clima en general en el largo plazo. Esto sugiere que los individuos multi-fustales constituyen una buena representación de la variabilidad del clima local. Debido a la gran extensión territorial de especie, otros estudios que abarquen la extensión geográfica de $P$. flexuosa son deseables para profundizar los resultados logrados.
\end{abstract}

Palabras clave: Algarrobo dulce, ancho de anillo, clima, variabilidad intra-especifica.

Summary: Tree growth habit differences as an explanatory variable of the climatic influence upon the radial growth variability of Prosopis flexuosa in the Central Monte Desert (Argentina). Dendroclimatological methods represent one of the most useful tools in analyzing the influence of climate upon the radial growth dynamics and morphology of woody species. In the Central Monte Desert, Prosopis flexuosa DC (algarrobo dulce), grows as one-stemmed as well as multi-stemmed tree. Previous researches showed the utility of the species ring widths as a proxy of climate variability. Nevertheless, these researches differed methodologically, by considering or rejecting the multi-stemmed trees. This work analyzed if the variability in the tree growth habit express differences in the algarrobo dulce dendroclimatological signal. Two tree-ring chronologies, one derived from the one-stemmed (UF) and the other from the multi-stemmed trees (MF) growing under the same ecological environment, were developed. The MF chronology showed higher sensibility than the UF to climate extremes and oscillation. This suggested that multi-stemmed trees represent a good proxy for explaining local climatic variability. Due to the widespread spatial distribution of this species, further works are needed to a deeper understanding of the presented results.

Key words: Algarrobo dulce, climate, intra-specific variability, ring width.

\footnotetext{
${ }^{1}$ Laboratorio de Dendrocronología e Historia Ambiental, IANIGLA, CCT-CONICET-Mendoza, Avda. Ruiz Leal s/n, Parque Gral. San Martín, CC 330, PO Box 5500 Mendoza, Argentina.

${ }^{2}$ E-mail: spiraino@mendoza-conicet.gov.ar
} 


\section{INTRODUCCIÓN}

Dentro de los roles ecosistémicos que posee la vegetación en zonas áridas y semi-áridas, se puede mencionar su función como sumidero de carbono, estructurador de ambientes, soporte de la vida silvestre, y como fuente de diversos servicios ecosistémicos (Puigdefábregas, 1998; Whitford, 2002). Este tipo de vegetación es altamente sensible a los cambios ambientales, en parte debido a las restricciones del recurso hídrico y a las elevadas tasas de evapo-transpiración que producen desequilibrios fisiológicos por estrés (Noy-Meir, 1973; Newman et al., 2006). Por ello, es de interés analizar la influencia que tienen los factores atmosféricos sobre la dinámica de crecimiento de las especies vegetales desérticas, particularmente asumiendo que la tensión clima-crecimiento puede alcanzar mayores niveles de estrés en contextos de potenciales modificaciones en el clima global (IPCC, 2007).

Los modelos biológicos basados en la relación entre la variabilidad de los anillos de crecimiento de los árboles y el clima (dendroclimatología: Kaennel \& Schweingruber, 1995) representan una útil herramienta en estudios sobre la dinámica de la vegetación leñosa, mejorando la comprensión sobre los modos en que los vegetales responden a la incidencia de diversos factores abióticos (Fritts, 1976). Asimismo, la relación entre incremento radial y factores climáticos no necesariamente es uniforme dentro de una población, pudiendo variar, por ejemplo, según la edad, el tamaño y el sexo de los individuos (Carrer \& Urbinati, 2004; De Luis et al., 2009; Hadad \& Roig, 2016).

En las zonas áridas y semi-áridas de Argentina, las especies del género Prosopis (algarrobo) son consideradas estructuradoras del ecosistema y representan un recurso alimentario y de hábitat fundamental para un gran número de organismos (Burkart, 1976; Roig, 1993a,b; Karlin et al., 1997). En el Desierto del Monte Central, Prosopis flexuosa DC, vulgarmente conocido como algarrobo dulce, domina el estrado arbóreo (Morello, 1958). En estos territorios, el algarrobo dulce presenta un alto nivel de plasticidad ecológica y fenotípica, expresado por la capacidad de la especie de crecer en territorios geomorfológicamente heterogéneos, y por la presencia en los mismos bosques de individuos uni y multi-fustales (Alvarez et al., 2006; Piraino et al., 2015).
Diferentes investigaciones han demostrado la utilidad del algarrobo dulce en análisis dendroclimatológicos, obteniendo informaciones de gran importancia para un mayor entendimiento de la influencia climática y ambiental sobre el crecimiento radial de la especie (Giantomasi et al., 2009, 2013; Piraino et al., 2015; Piraino \& Roig, 2016). Sin embargo, en dichas investigaciones ha habido diferencias en aspectos metodológicos, ya que, por ejemplo, la consideración de individuos unio multi-fustales no ha tenido el mismo tratamiento de análisis. Piraino et al. (2015) desarrollaron cronologías de ancho de anillos incluyendo ambas categorías morfológicas, mientras que Giantomasi et al. (2013) sugieren descartar los individuos multi-fustales, ante la posibilidad de que sean consecuencia de disturbios, y por consiguiente, aporten efectos de variabilidad sobre el crecimiento radial que no están vinculados al clima. Aún cuando se discute respecto de incluir información derivada de individuos multi-fustales en estudios locales, el tema alcanza a otras investigaciones donde individuos multi-fustales han sido evitados en análisis dendroclimatológicos (Wang et al., 2005; Gareca et al., 2010; Bowman et al., 2011). Por lo previamente expuesto, cabe preguntarse entonces: ¿los algarrobos que difieren por sus características morfológicas presentan variaciones intra-especificas en la relación entre el ancho de los anillos y clima?; si se cumple dicho supuesto, ¿cual es la calidad de la información derivada de individuos multi-fustales en interpretaciones dendroclimatológicas? Por ello, en este trabajo proponemos analizar la variabilidad del ancho de los anillos de P. flexuosa y su vinculación con los factores climáticos en individuos tanto uni- como multi-fustales.

\section{Materiales y Métodos}

\section{Obtención de las muestras}

Los muestreos se llevaron a cabo en el Desierto del Monte Central correspondiente al NE de la Provincia de Mendoza. Según la clasificación climatológica de Köppen-Geiger, esta área es definida como un ambiente semi-árido (BSh), con precipitación anual alrededor de $155 \mathrm{~mm}$ y elevada variabilidad estacional y diaria en valores de temperatura (Peel et al. 2007). El sitio de muestreo 
(32 ${ }^{\circ} 18^{\prime} 51,99^{\prime}$ LS, $67^{\circ} 38^{\prime} 18,1$ ' LO; Fig. 1) está caracterizado por un bosque mono-especifico de $P$. flexuosa instalado sobre suelo franco-arenoso (arena $=50 \%$; limo $=34 \%$; arcilla $=17 \%)$ en ambiente de paleo-cauce (Rubio et al. 2009). El bosque analizado posee una limitada extensión espacial, de aprox. $2000 \mathrm{~m}^{2}$, y es localizado en una extensa planicie de suelo desnudo.

En este bosque se delimitó una parcela de 1000 $\mathrm{m}^{2}(50 \mathrm{~m} \times 20 \mathrm{~m})$ en la que se midieron todos los individuos cuyo diámetro a altura de pecho (DAP) fuera $>7,5 \mathrm{~cm}$ (de acuerdo con Alvarez et al. 2006). Para cada individuo se registró el DAP de tronco y la altura de copa. En el caso de los algarrobos multi-fustales, se registró el DAP de cada fuste. Posteriormente, de cada árbol se extrajeron dos muestras con barreno mecanizado y broca de 1,2 $\mathrm{cm}$ de diámetro a una altura de aproximadamente $50 \mathrm{~cm}$ sobre el nivel del suelo. Para los individuos multi-fustales se muestreó sólo la madera del fuste principal (identificado como el de mayor DAP). El muestreo dendrocronológico incluyó un total de 26 individuos, 14 uni-fustales y 12 multi-fustales.

\section{Construcción de las cronologías}

Las muestras fueron montadas, pulidas con lijas de granulometría ascendente (desde 60 hasta 1000), fechadas y escaneadas con resolución de 1200 dpi (escáner EPSON Perfection V500 Photo). La medición del ancho de los anillos fue realizada a través del programa IPWin4 (v4.5, Media Cybernetics, EEUU), y la calidad de las mediciones fue evaluada estadísticamente mediante el programa COFECHA (Holmes, 1983). Posteriormente, las series individuales de ancho de anillos fueron estandardizadas (Fritts, 1976) con el objetivo de remover la señal vinculada a la tendencia biológica en el crecimiento radial y/o determinados eventos de disturbio (representado en el área examinada por la tala de P. flexuosa: Piraino et al., 2017). El proceso de estandardización se llevó a cabo aplicando a cada serie dendrocronológica individual una curva exponencial negativa y luego una curva polinomial de tercer grado de 32 años de extensión y con retención del 50\% de la variabilidad (Cook, 1987). Los cálculos fueron realizados mediante el software ARSTAN40c (Cook \& Krusic, 2006).
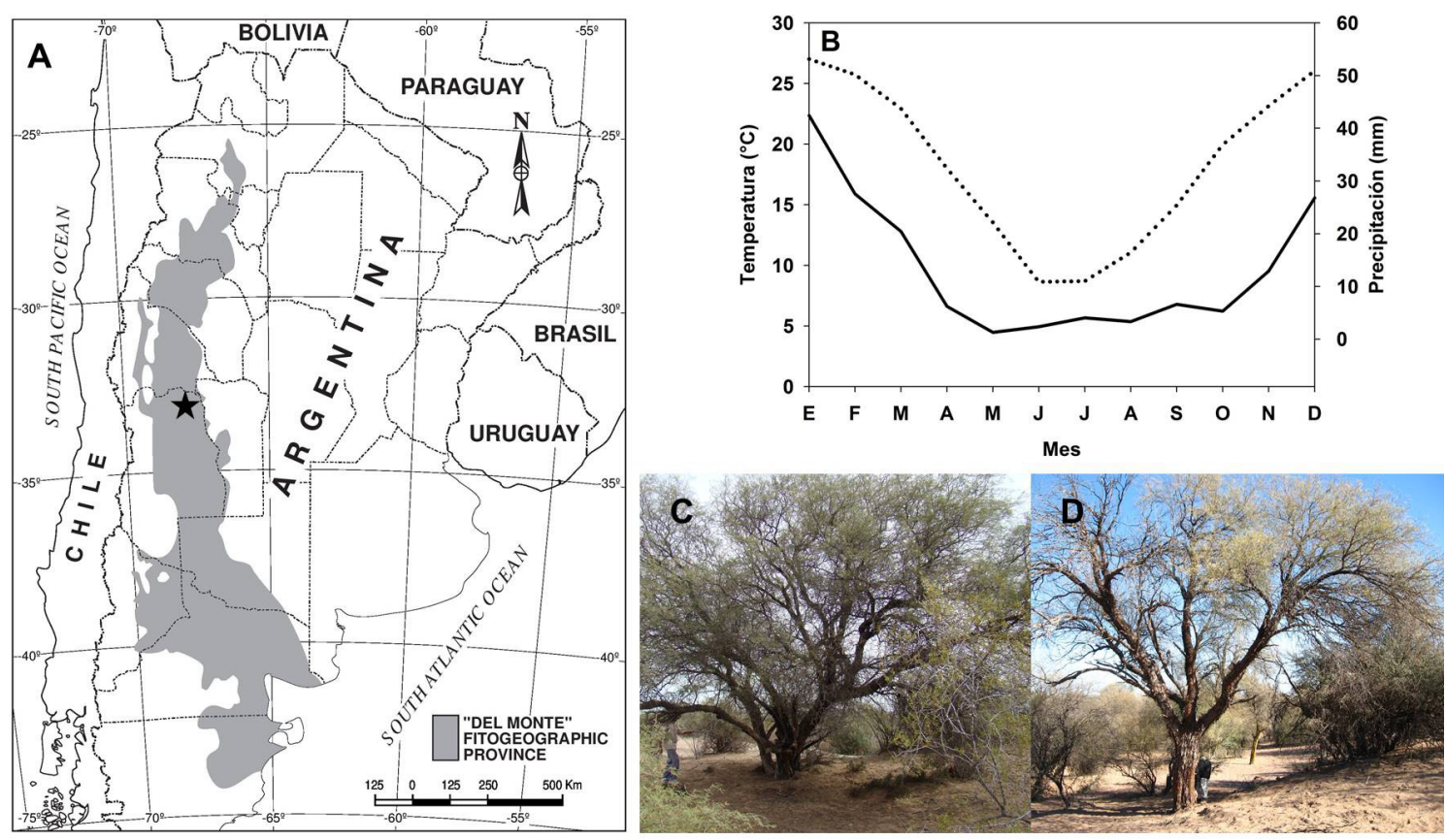

Fig. 1. Área de estudio (A). La estrella índica el sitio de muestreo. Diagrama ombrotérmico según Bagnouls \& Gaussen (1953) para la estación meteorológica de Encón (32 15’ S, 67 47’ O., 521 msnm) (B) donde: (i) la línea continua representa los valores de precipitación total mensual $(\mathrm{mm})$, y (ii) la línea puntuada expresa los valores de temperatura media mensual $\left({ }^{\circ} \mathrm{C}\right)$. Ejemplos de algarrobo dulce multi-fustal (C) y uni-fustal (D). 
La calidad de las cronologías fue evaluada considerando dos índices estadísticos, el EPS y el RBAR (Wigley et al., 1984). El EPS representa una estimación sobre el número mínimo de muestras necesarias para considerar estadísticamente confiable una cronología de ancho de anillos, mientras que el RBAR corresponde al valor promedio de las correlaciones entre todas las series que contribuyen a la construcción de la cronología del sitio examinado (Wigley et al, . 1984). Tanto EPS como RBAR fueron calculados utilizando ventanas móviles de 20 años con solapamiento de 19 años. Finalmente, los valores de ambos índices fueron contrastados con los obtenidos para $P$. flexuosa en otros registros dendrocronológicos (Giantomasi et al., 2013; Piraino et al., 2015; Piraino \& Roig, 2016).

\section{Análisis estadísticos}

Con el objetivo de identificar posibles diferencias intra-especificas en la relación entre el clima y el crecimiento radial, y que estos fuesen función de las características del hábito de crecimiento, se realizaron análisis de la varianza simples no-paramétricos (prueba de KruskalWallis) considerando como variables los datos dasonómicos (DAP y altura) y la edad de los individuos por conteo de anillos de crecimiento, y como factores de análisis las morfologías de los arboles muestreados (uni- vs. multi-fustales). Los análisis fueron efectuados mediante INFOSTAT/P (2008).

\section{Relación entre ancho de anillo y factores climáticos}

La vinculación entre el crecimiento radial y las fluctuaciones en el régimen climático fue analizada considerando como variable dependiente el crecimiento (bruto y estandarizado) y como variables independientes los valores (anuales y mensuales) de precipitación total y temperatura media, ambas obtenidas de la estación meteorológica del Aeropuerto de San Juan (31 $34^{\circ}$ LS; 68 $25^{\circ}$ LO).

En la búsqueda de estas interacciones climacrecimiento, inicialmente se procedió al cálculo de los "años característicos", definidos como aquellos años en los que un determinado porcentaje de individuos responden a condiciones particularmente favorables o adversas con cambios abruptos positivos o negativos en el crecimiento radial no-estandardizado (Schweingruber et al., 1990). Estos años característicos fueron computados adoptando el método propuesto por Cropper (1979) y mediante el programa WEISER (Gonzalez, 2001), seleccionando una ventana temporal de 5 años para definir como característicos aquellos años en los cuales por lo menos el $80 \%$ de los individuos analizados muestran un cambio en el crecimiento radial (positivo o negativo) mayor al $50 \%$. Luego, con el objetivo de analizar la posible influencia abiótica sobre estos cambios abruptos en el crecimiento, los años característicos fueron comparados con los valores de precipitación y temperatura media anual (calculados para el periodo 1973-2011), correspondientes al promedio del periodo que va desde el mes abril del año previo hasta marzo del año corriente del crecimiento.

Posteriormente y mediante funciones de correlación simple (Blasing et al., 1984), se compararon las cronologías estandardizadas de ancho de anillos y los valores mensuales de precipitación total y temperatura media. Se seleccionó una ventana de 12 meses, desde abril del año previo al crecimiento vegetativo hasta marzo del año corriente. Las funciones de correlación fueron computadas a través del programa DENDROCLIM2002, considerando el método bootstrap que minimiza la co-linealidad entre datos climáticos (Guiot, 1991; Biondi \& Waikul, 2004). En todos los cálculos se estableció como periodo de análisis el intervalo 1981-2010, en el que no se produjeron episodios de tala en el bosque analizado (Piraino et al., datos no publicados).

\section{Resultados}

Características dasonómicas y dendrocronológicas

Los individuos multi-fustales mostraron valores más elevados en relación con el DAP de los algarrobos uni-fustales, mientras que la altura y la edad alcanzaron mayores valores en éstos últimos (Tabla 1). Sin embargo, ninguna de estas diferencias resultó ser estadísticamente significativa (Fig. 2).

Veintiséis muestras dendrocronológicas, correspondientes a un total de 21 individuos, fueron empleadas para construir las dos cronologías de ancho de anillos de árboles uni-fustales (UF) y multi-fustales (MF). Las cronologías presentaron una extensión temporal equivalente entre UF 
Tabla 1. Características promedio dasonómicas y etarias de los árboles analizados según el hábito de crecimiento. Entre paréntesis se indican los valores mínimos y máximos.

\begin{tabular}{|cccc|}
\hline Hábito de crecimiento & Diámetro a altura de pecho $(\mathrm{cm})$ & Altura $(\mathbf{m})$ & Edad (años) \\
\hline Unifustal & $12,95(10,00-18,40)$ & $5,44(3,50-9,00)$ & $45(28-80)$ \\
Multifustal & $13,61(10,00-16,00)$ & $5,40(3,50-7,00)$ & $40(25-79)$ \\
\hline
\end{tabular}

y MF (Tabla 2), de 40-45 años. El incremento anual promedio resultó ser mayor en la cronología MF respecto de la UF (Tabla 2). Los valores de correlación entre series fueron similares y ambos estadísticamente significativos al nivel $P<0,05$ (Tabla 2). En relación a los índices EPS y RBAR (Tabla 2), ambos resultaron ser mayores en la cronología MF $(0,80$ y 0,35$)$ respecto a la UF $(0,76$ y 0.34$)$, y se encuentran en el rango de valores conocido para la especie examinada (Tabla 3).

\section{Relación entre clima y crecimiento radial}

El análisis de los cambios abruptos en el crecimiento bruto permitió detectar respectivamente uno (cronología UF) y cuatro (MF) años característicos. En relación a los árboles unifustales, se identificó un anillo angosto para el año 1988 y ocurrente en el $80 \%$ de los individuos analizados (Tabla 4). Por otro lado, la cronología MF presentó dos valores positivos sobresalientes en los años 1987 (82\% de los árboles analizados) y 2010 (83\%), mientras que los anillos angostos fueron detectados en los años 1994 y 2008 , en el $91 \%$ y el $86 \%$ de los individuos multi-fustales analizados, respectivamente (Tabla 4). Finalmente, la comparación entre valores anuales de los factores climáticos y cambios en el crecimiento indica que los años característicos positivos (negativos) dependen en mayor medida del efecto de abundantes (escasas) precipitaciones, siendo menos marcada la influencia de las temperaturas anuales sobre estos máximos y mínimos en el incremento radial bruto (Tabla 4).

Los resultados obtenidos mediante las correlaciones evidencian diferencias entre las señales dendroclimatológicas obtenidas para las cronologías UF y MF, respectivamente (Fig. 3). El ancho del anillo relativo a la cronología UF se encuentra influido de forma positiva por la variabilidad en la precipitación del mes de marzo del año de crecimiento $(r=0,29 ; P<0,05)$, mientras que en la cronología MF se evidenció una relación positiva entre el crecimiento radial y las lluvias de los meses de octubre del año previo al crecimiento $(r=0,26 ; P<0,05)$ y febrero del año corriente $(r=0,27 ; P<0,05$; Fig. 3). En relación a la influencia de las temperaturas, no se obtuvo resultado estadísticamente significativo para ambas cronologías.

\section{Discusión}

La ausencia de diferencias estadísticamente significativas en relación a las características dasonómicas y etarias entre los árboles analizados sugiere que la variabilidad dendroclimatológica encontrada en este estudio es, muy probablemente, función de la heterogeneidad intra-específica del hábito de crecimiento debido a los diferentes tipos morfológicos. Generalmente, la presencia de múltiples fustes en una planta se relaciona con la capacidad de rebrotar luego de un evento de disturbio (Bellingham \& Sparrow, 2000). Esto a la vez puede implicar para el individuo un costo fisiológico relacionado con el almacenamiento de azucares y la protección de un banco de yemas (Vesk \& Westoby, 2004). Por otro lado, la presencia de múltiples fustes puede generar procesos de competencia entre fustes de un mismo árbol. En este sentido, Cao \& Peters (1998) sugirieron que en los individuos multi-fustales del haya china (Fagus engleriana Seemen ex Diels), los distintos fustes compiten por los recursos limitantes al crecimiento radial (agua y nutrientes). En Lindera triloba (Siebold \& Zuccarini) Blume se ha observado que la competencia por los recursos (hidratos de carbono, nutrientes) entre fustes de un mismo individuo afecta negativamente el crecimiento y la supervivencia de esta planta (Matsushita et al., 2010). También se ha visto que el crecimiento de individuos multifustales de distintas especies arbóreas de bosques 
Bol. Soc. Argent. Bot. 52 (3) 2017

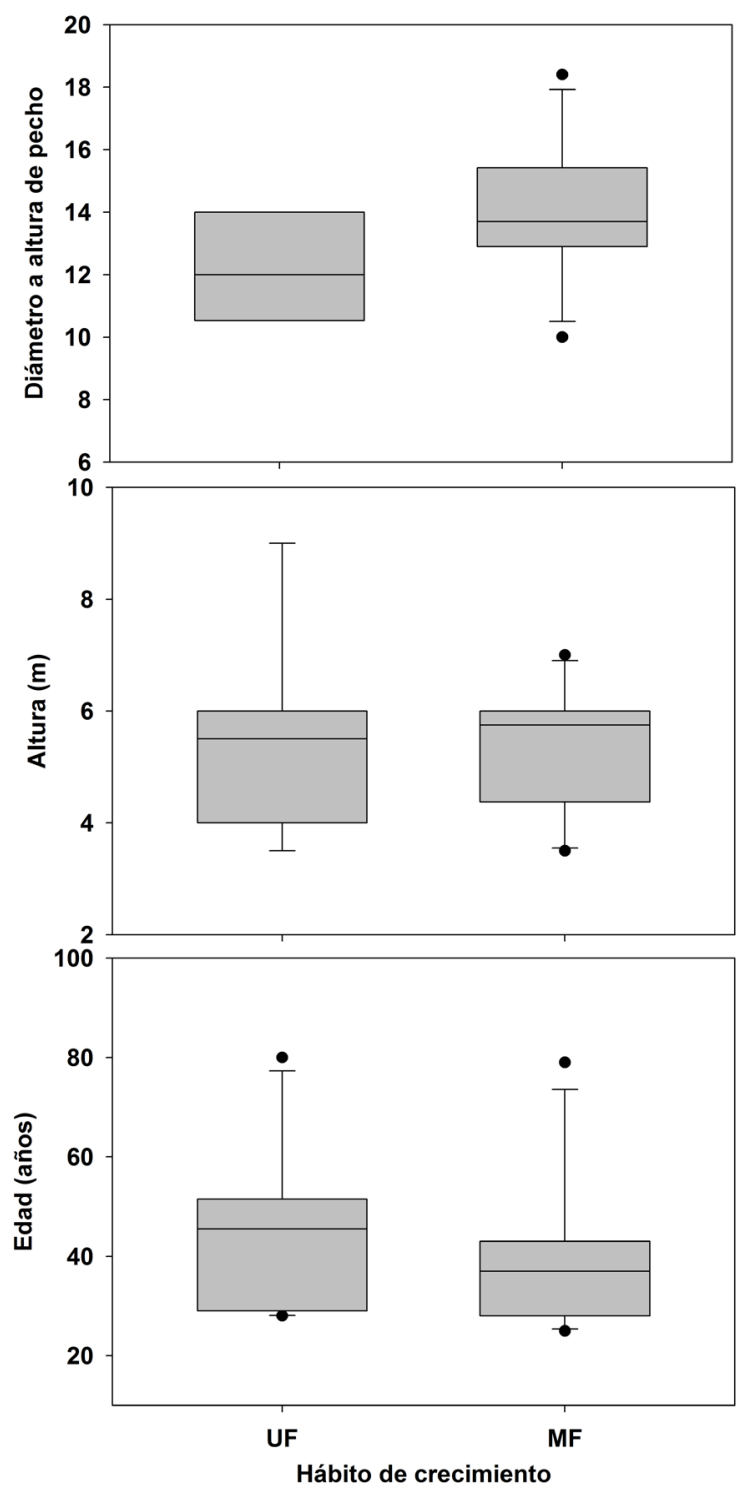

Fig. 2. Gráficos de caja-bigote para los valores de diámetro a altura de pecho (arriba), altura (medio) y edad (abajo) para los individuos analizados según el hábito de crecimiento. Cada caja muestra los valores comprendido en la distancia de un intercuartil ( $25 \%$ por encima y por debajo del promedio). El promedio se muestra como línea negra. Los bigotes corresponden a un valor de 1.5 veces la distancia de un intercuartil, y se muestran como líneas negras. Los círculos negros representan los valores atípicos.

templados boreales se ve afectado negativamente por el aumento en el número de los fustes, lo que es atribuido a fenómenos de competencia (Tanentzap et al., 2011). En conjunto entonces, las distintas formas de hábito de crecimiento pueden traducirse en diferencias fisiológicas, lo cual podría explicar las distintas sensibilidades de algarrobos uni- y multifustales a la variabilidad climática detectadas en esta investigación.

Los relativamente altos valores de correlación entre series individuales y dentro de la población examinada indican la presencia de un importante 
Tabla 2. Características de las cronologías de ancho de anillo. N: número de árboles series que componen cada cronología (entre paréntesis: número de series). AA: valor promedio del ancho de anillo. CES: correlación entre series. EPS y RBAR: valores promedio de los dos índices.

\begin{tabular}{|ccccccc|}
\hline Cronología & $\mathbf{N}$ & Periodo & AA $(\mathbf{m m})$ & CES & EPS & RBAR \\
UF & $10(13)$ & $1931-2010$ & 1,36 & 0,48 & 0,76 & 0,34 \\
MF & $11(13)$ & $1932-2010$ & 1,74 & 0,49 & 0,80 & 0,35 \\
\hline
\end{tabular}

porcentaje de variabilidad común, lo cual sugiere que un mismo factor ambiental podría estar influyendo de forma preponderante sobre el crecimiento de los individuos. Esto está vinculado al alto valor expresado por los índices EPS y RBAR, en comparación con resultados derivados de estudios previos (Tabla 3). En este sentido, los índices aquí presentados alcanzaron los valores más elevados considerando los algarrobales pertenecientes al Desierto del Monte Central, siendo de hecho los EPS solo inferiores a los encontrados por Giantomasi et al. (2013) en relación a los bosques de P. flexuosa instalados en el SE del Chaco Árido. Por otro lado, hay que subrayar que los valores de RBAR obtenidos a partir de las cronologías aquí desarrolladas son los más altos obtenidos para la especie en su entera área de distribución geográfica (Giantomasi et al., 2013;

Tabla 3. Comparación de los valores promedio de EPS y RBAR para $P$. flexuosa presentes en la literatura dendroclimatológica sobre la especie.

\begin{tabular}{|cccc|}
\hline Cronología & EPS & RBAR & Fuente \\
MF & 0,80 & 0,35 & Este trabajo \\
UF & 0,76 & 0,34 & Este trabajo \\
Arid Chaco & 0,94 & 0,33 & Giantomasi et al. (2013) \\
Monte & 0,78 & 0,23 & Giantomasi et al. (2013) \\
Transition & 0,91 & 0,24 & Giantomasi et al. (2013) \\
Ceferino & 0,71 & 0,22 & Piraino et al. (2015) \\
El Mateo & 0,72 & 0,18 & Piraino et al. (2015) \\
La Juana & 0,75 & 0,22 & Piraino et al. (2015) \\
La Toma & 0,65 & 0,25 & Piraino et al. (2015) \\
Pozo Verde & 0,70 & 0,17 & Piraino et al. (2015) \\
Puesto Viejo & 0,70 & 0,22 & Piraino et al. (2015) \\
Río Viejo & 0,80 & 0,26 & Piraino et al. (2015) \\
EMF & 0,58 & 0,12 & Piraino \& Roig (2016) \\
\hline EMN & 0,74 & 0,16 & Piraino \& Roig (2016) \\
\hline
\end{tabular}

Piraino et al., 2015; Piraino \& Roig, 2016). Además de reflejar una buena sincronía entre árboles, los valores de EPS y RBAR confieren a las cronologías aquí desarrolladas confiabilidad estadística y por ende sobre los resultados dendrocronológicos obtenidos.

En relación a la productividad medida como incremento radial del xilema, los individuos multi-fustales presentaron un valor promedio de crecimiento mayor respecto a los de fuste único $(1,74$ vs. $1,36 \mathrm{~mm} / \mathrm{anno}$ ). Considerando que las cronologías aquí desarrolladas están compuestas por individuos cuya edad promedio es de 40/45 años, este resultado es coherente con lo encontrado por Álvarez et al. (2011), quienes evidenciaron que, hasta los 60 años de edad, los algarrobos de múltiples fustes presentan mayor incremento diamétrico respecto de los de un solo fuste. Esto es argumentado como consecuencia de una mayor superficie foliar y su influencia sobre la actividad fotosintética (Álvarez et al. 2011).

$\mathrm{Al}$ efectuar las comparaciones entre la variabilidad del clima y el crecimiento, el análisis del incremento radial bruto evidenció una mayor sensibilidad en la

Tabla 4. Comparación entre años característicos para las cronologías UF y MF y valores anuales

promedio del clima obtenidos de la estación meteorológica del Aeropuerto de San Juan. Los valores de precipitación y temperatura se refieren al porcentaje respecto del promedio histórico por el periodo 1973-2011.

\begin{tabular}{|ccccc|}
\hline & UF & MF & $\begin{array}{c}\text { Precipitación } \\
\text { anual }\end{array}$ & $\begin{array}{c}\text { Temperatura } \\
\text { anual }\end{array}$ \\
\hline 1987 & & + & $106 \%$ & $100 \%$ \\
1988 & - & & $51 \%$ & $99 \%$ \\
1994 & & - & $13 \%$ & $102 \%$ \\
2008 & - & $93 \%$ & $104 \%$ \\
2010 & + & $450 \%$ & $99 \%$ \\
\hline
\end{tabular}


Bol. Soc. Argent. Bot. 52 (3) 2017

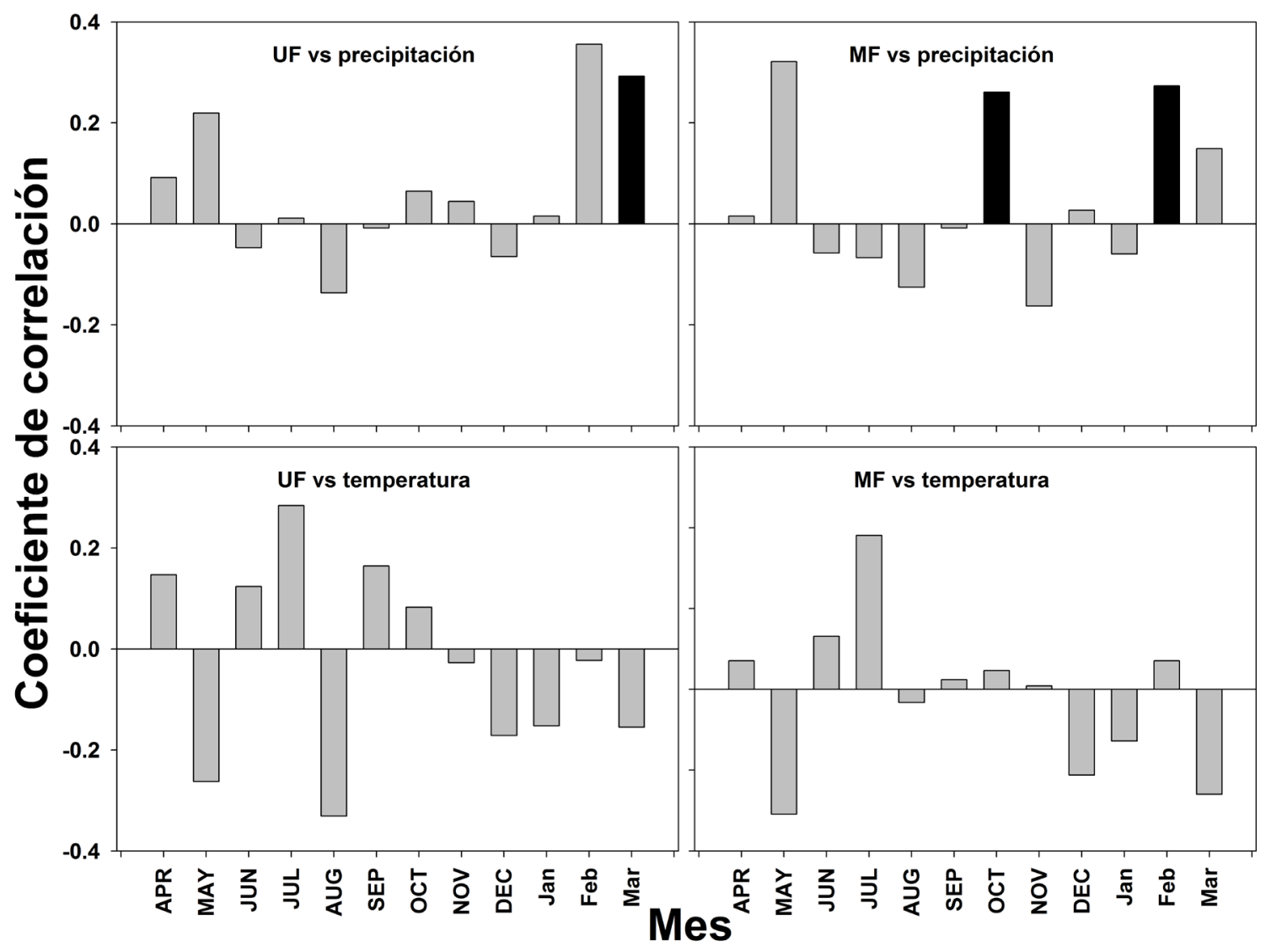

Fig. 3. Gráfico de barra del coeficiente de correlación entre las cronologías residuales UF y MF y los valores mensuales de precipitación y temperatura promedio de la estación meteorológica del Aeropuerto de San Juan. Las barras negras indican valores estadísticamente significativos por $p<0,05$ entre cronologías y factores climáticos.

reacción del crecimiento de los individuos múltifustes ante años particularmente lluviosos y secos. Esto es ejemplificado, por un lado, por la mayor cantidad de años característicos registrados en la cronología MF (4) respecto de la UF (1). Por otro lado, más importante aún parece ser el resultado inherente a la respuesta de la cronología MF a dos años (1994 y 2010) en lo que llovió el 13\% y el $450 \%$ respecto al promedio histórico relativo al periodo 1973-2011, y de los que emergieron años característicos negativos y positivos, a diferencia de lo observado en la cronología UF. Este resultado confirma la elevada variabilidad intra-especifica en el crecimiento radial bruto del algarrobo dulce en relación a los máximos y mínimos climáticos (Piraino et al., datos no publicados).
Las funciones de correlación indicaron que la precipitación es el factor abiótico más importante para el desarrollo del ancho del anillo, en coincidencia con lo indicado en estudios previos sobre la dendroclimatología de P. flexuosa en el Desierto del Monte Central (Piraino, 2016). El cálculo de las funciones de correlación indicó, al igual que lo encontrado a través de los años característicos, una diferencia en la señal dendroclimatológica atestiguada particularmente por la ausencia para la cronología UF de la influencia sobre el crecimiento de las precipitaciones de octubre.

Biológicamente, los resultados obtenidos a partir de las funciones de correlación pueden entenderse considerando las diferentes fases de formación del anillo anual en P. flexuosa, que en 


\section{S. Piraino y F. A. Roig Juñent - Influencia climática en Prosopis flexuosa en Argentina}

el Desierto del Monte Central tienen lugar en el periodo comprendido entre octubre del año previo del crecimiento hasta marzo del año corriente (Giantomasi et al., 2012). En este sentido, la relación positiva entre la precipitación de octubre del año previo del crecimiento y la cronología MF, sugiere que en los individuos multi-fustales existe una dependencia del ancho del anillo a factores atmosféricos vinculados al principio de la temporada de crecimiento. Por otro lado, los restantes resultados indican un vínculo directo entre el incremento radial con valores de precipitación situados por encima del valor medio histórico para los meses correspondientes a finales de la temporada de crecimiento, tanto para la cronología UF (marzo) como para la MF (febrero). Esto se debe a que en el algarrobo dulce, aún cuando a principios de enero están dadas las condiciones para que el anillo de crecimiento concluya el desarrollo de su madera tardía, entre fin de enero y marzo puede producirse un segundo pulso de incremento xilemático, en correspondencia a condiciones climáticas favorables definidas por la acción combinada de temperaturas del aire menores y de pulsos de precipitación (Giantomasi et al., 2012).

Estudios previos indican que para los bosques de P. flexuosa instalados en ambiente de paleo-cauce en el Desierto del Monte Central, el ancho del anillo se encuentra beneficiado por lluvias abundantes hacia el final del primer pulso de crecimiento y al principio del segundo (Piraino et al., 2015). En este sentido, nuestros resultados confirman, en el caso de ambas cronologías, la importancia de la precipitación de final de verano en el valor del incremento radial del algarrobo dulce. Sin embargo, la relación entre el anillo de crecimiento y las precipitaciones del mes de octubre detectada para la cronología MF podría estar, según investigaciones previas, más vinculada a unidades geomorfológicas correspondientes a ambientes de mayor xericidad, como por ejemplo los sitios de médano y planicie con depósito arcillo-limoso (Piraino et al., 2015). Esto sugiere entonces que los algarrobos multifustales podrían encontrarse en una situación de estrés hídrico mayor respecto a los de un solo fuste, lo cual justificaría además la mayor sensibilidad de los valores máximos y mínimos en el crecimiento bruto de la cronología MF a años particularmente lluviosos y/o secos, como surge del cálculo de los años característicos.
No existen antecedentes bibliográficos sobre si el hábito de crecimiento modula la respuesta al clima de una especie arbórea desértica. A pesar de esto Devi et al. (2009) examinó la dinámica del límite superior del bosque en los Monte Urales (Rusia) donde encontraron que los alerces siberianos (Larix sibirica L.) de un solo fuste presentaban una mayor vinculación entre el crecimiento y los valores de precipitación de verano e invierno respecto de individuos multi-fustales. Este resultado podría ser sesgado, sin embargo, por la menor edad de los alerces uni-fustales, bajo la hipótesis de que en dicha especie una respuesta del crecimiento radial a los factores climáticos es dependiente de la edad (Yu et al., 2008).

Finalmente, los resultados logrados permiten indicarquelosindividuosmulti-fustales de P.flexuosa aportan valiosa información dendroclimática de los bosques en el Desierto del Monte Central. Las evidencias aquí presentadas sugieren una mayor sensibilidad de los individuos multi-fustales a las variables condiciones meteorológicas. Cambios en valores de precipitación para la zona centro-oeste de Argentina indicados por proyecciones climáticas (Labraga \& Villalba, 2009), podrían potencialmente incidir en la estructura y dinámica natural de estos algarrobales, poniendo en relieve los resultados aquí obtenidos en estimaciones futuras sobre la conservación de los bosques del Desierto del Monte Central.

\section{Agradecimientos}

El primer autor agradece CONICET por una beca post-doctoral interna. Muchas gracias a la familia Agüero y a la Dirección de Recursos Naturales Renovables de la Provincia de Mendoza por autorizar los muestreos, y a Alberto Ripalta (IANIGLA CCT-Mendoza) por su ayuda en el trabajo de campo. Un agradecimiento especial al revisor anónimo y a sus comentarios que mejoraron sensiblemente la calidad de este manuscrito.

\section{Bibliografía}

AlvareZ, J. A., P. VILlagra, M. A. CONY, E. M. CESCA, \& J. A. BONINSEGNA. 2006. Estructura y estado de conservación de los bosques 
de Prosopisflexuosa D.C. (Fabaceae, subfamilia: Mimosoideae) en el noreste de Mendoza (Argentina). Rev. Chil. Hist. Nat. 79: 75-87.

ALVAREZ, J. A., P. VILLAGRA, R. VILLALBA, M. A. CONY, \& M. ALBERTO. 2011. Wood productivity of Prosopis flexuosa DC woodlands in the central Monte: influence of population structure and treegrowth habit. J. Arid Environ. 75: 7-13.

BAGNOULS, F., \& H. GAUSSEN. 1953. Saison sèche et indice xérothermique. Bull. Soc. hist. nat. Toulouse 88: 193-240.

BELLINGHAM, P. J., \& A. D. SPARROW. 2000. Resprouting as a life history strategy in woody plant communities. Oikos 89: 409-416.

BIONDI, F., \& K. WAIKUL. 2004. DENDROCLIM2002: A $\mathrm{C}++$ program for statistical calibration of climate signals in tree-ring chronologies. Comput. Geosci. 30: 303-311.

BLASING, T. J., A. M. SOLOMON \& D.N. DUVICK. 1984. Response functions revisited. Tree-Ring Bull. 44: 1-15.

BOWMAN, D. M., L. D. PRIOR, D. Y. TNG, Q. HUA, \& T. J. BRODRIBB. 2011. Continental-scale climatic drivers of growth ring variability in an Australian conifer. Trees 25: 925-934.

BURKART, A. 1976. A monograph of the genus Prosopis (Leguminosae subfam. Mimosoideae). J. Arnold Arboretum 57: 450-455.

CARRER, M., \& C. URBINATI. 2004. Age-dependent tree-ring growth responses to climate in Larix decidua and Pinus cembra. Ecology 85: 730-740.

CAO, K. F., \& R. PETERS. 1998. Structure and stem growth of multi-stemmed trees of Fagus engleriana in China. Plant Ecol. 139: 211-220.

COOK, E. R. 1987. The decomposition of tree-ring series for environmental studies. Tree-Ring Bull. 47: 37-59.

COOK, E. R., \& P .J. KRUSIC. 2006. ARSTAN 41: a tree-ring standardization program based on detrending and autoregressive time series modeling, with interactive graphics. Tree-Ring Laboratory, Lamont Doherty Earth Observatory of Columbia University, New York.

CROPPER, J. P. 1979. Tree-ring skeleton plotting by computer. Tree-Ring Bull. 39: 47-59.

DE LUIS, M., K. NOVAK, K. ČUFAR, \& J. RAVENTÓS. 2009. Size mediated climate-growth relationships in Pinus halepensis and Pinus pinea. Trees 23: $1065-$ 1073.

DEVI, N., F. HAGEDORN, P. MOISEEV, H. BUGMANN, S. SHIYATOV, V. MAZEPA, \& A. RIGLING. 2008. Expanding forests and changing growth forms of Siberian larch at the Polar Urals treeline during the 20th century. Global Change Biol. 14: 1581-1591.

FRITTS H.C. 1976. Tree Rings and Climate. Academic Press, London.
GARECA, E. E., M. FERNÁNDEZ, \& S. STANTON. 2010. Dendrochronological investigation of the high Andean tree species Polylepis besseri and implications for management and conservation. Biodivers. Conserv. 19: 1839-1851.

GIANTOMASI, M. A., F. A. JUNENT, P. E. VILLAGRA, \& A. M. SRUR. 2009. Annual variation and influence of climate on the ring width and wood hydrosystem of Prosopis flexuosa DC trees using image analysis. Trees 23: 117-126.

GIANTOMASI, M. A., F. A. ROIG-JUÑENT, D. PATÓN-DOMÍNGUEZ, \& G. MASSACCESI. 2012. Environmental modulation of the seasonal cambial activity in Prosopis flexuosa DC trees from the Monte woodlands of Argentina. J. Arid Environ. 76: 17-22.

GIANTOMASI, M. A., F. A. ROIG-JUÑENT, \& P. E. VILLAGRA 2013. Use of differential water sources by Prosopis flexuosa DC: A dendroecological study. Plant Ecol. 214: 11-27.

GONZALEZ, I. G. 2001. Weiser: a computer program to identify event and pointer years in dendrochronological series. Dendrochronologia 19: 239-244.

GUIOT, J. 1991. The bootstrapped response function. Tree-Ring Bull. 51: 39-41.

HADAD, M. A., \& F. A. ROIG-JUÑENT. 2016. Sexrelated climate sensitivity of Araucaria araucana Patagonian forest-steppe ecotone. Forest Ecol. Manage. 362: 130-141.

HOLMES, R. L. 1983. Computer-assisted quality control in tree-ring dating and measurement. Tree-Ring Bull. 43: 69-78.

INFOSTAT/P. 2008. Infostat profesional. UNC, FCA, Córdoba, Argentina.

IPCC WG I, 2007. Climate Change: The Physical Science Basis. Contribution of Working Group I. En: SOLOMON S., QIN D, MANNING M., CHEN Z., MARQUIS M., AVERYT K.B., TIGNOR M., \& H.L. MILLER (Eds.), Fourth Assessment Report of the Intergovernmental Panel on Climate Change. Cambridge University Press, Cambridge.

KAENNEL, M., \& F. H. SCHWEINGRUBER. 1995. Multilingual glossary of dendrochronology. Paul Haupt AG Bern.

KARLIN, U., R. COIRINI, L. CATALÁN, \& R. ZAPATA. 1997. Prosopis flexuosa. En: Especies arbóreas y arbustivas para las Zonas Áridas y Semiáridas de América Latina. Serie Zonas Áridas y Semiáridas. FAO, Santiago, Chile, 12: 51-61.

LABRAGA, J. C., \& R. VILLALBA. 2009. Climate in the Monte Desert: past trends, present conditions, and future projections. J. Arid Environ. 73: 154-163.

MATSUSHITA, M., N. TOMARU, D. HOSHINO, N. NISHIMURA, \& S. I. YAMAMOTO. 2010. Factors 


\section{S. Piraino y F. A. Roig Juñent - Influencia climática en Prosopis flexuosa en Argentina}

affecting the production, growth, and survival of sprouting stems in the multi-stemmed understory shrub Lindera triloba. Botany 88: 174-184.

MORELLO, J. 1958. La Provincia Fitogeográfica del Monte. Tucumán: Opera Lilloana II, 155.

NEWMAN, B. D., B. P. WILCOX, S. R. ARCHER, D. D. BRESHEARS, C. N. DAHM, C. J. DUFFY, MCDOWELL, N. G., PHILLIPS, F. M., SCANLON, B. R., \& E. R. VIVONI. 2006. Ecohydrology of water-limited environments: A scientific vision. Water Resour. Res. 42: [insertar níumeros de página].

NOY-MEIR, I. 1973. Desert ecosystems: environment and producers. Annu. Rev. Ecol. Syst. 4: 25-51.

PEEL, M. C., B. L. FINLAYSON, \& T. A. MCMAHON. 2007. Updated world map of the Köppen-Geiger climate classification. Hydrol. Earth Syst. Sci. 11: 1633-1644.

PIRAINO, S., E. M. ABRAHAM, A. DIBLASI, \& F. A. ROIG-JUÑENT. 2015. Geomorphological-related heterogeneity as reflected in tree growth and its relationships with climate of Monte Desert Prosopis flexuosa DC woodlands. Trees 29: 903-916.

PIRAINO, S. 2016. Influencia de la heterogeneidad ambiental y el disturbio sobre la dinámica en el crecimiento radial de Prosopis flexuosa DC en el Desierto del Monte Central. Ecosistemas 25: $105-$ 107.

PIRAINO, S., \& F. A. ROIG-JUÑENT. 2016. Assessing the sensitivity of riparian algarrobo dulce (Prosopis flexuosa DC) radial growth to hydrological changes. Geochronometria 43: 1-8.

PIRAINO, S., E. M. ABRAHAM, M. A. HADAD, D. PATÓN, \& F. A. ROIG-JUÑENT. 2017. Anthropogenic disturbance impact on the stem growth of Prosopis flexuosa DC forests in the Monte desert of Argentina: A dendroecological approach. Dendrochronologia 42: 63-72.

PUIGDEFÂBREGAS, J. 1998. Ecological impacts of global change on drylands and their implications for desertification. Land Degrad. Dev. 9: 393-406.

ROIG, F. A. 1993a. Informe nacional para la Selección de Germoplasma en Especies del Género Prosopis de la República Argentina. En: Contribuciones Mendocinas a la Quinta Reunión Regional para América Latina y el Caribe de la Red de Forestación del CIID. Conservación y Mejoramiento de Especies del Género Prosopis. IADIZA, Mendoza, Argentina, pp 1-36.
ROIG, F. A. 1993b. Aportes a la etnobotánica del género Prosopis. En: Contribuciones Mendocinas a la Quinta Reunión de Regional para América Latina y el Caribe de la Red de Forestación del CIID. Conservación y Mejoramiento de Especies del Género Prosopis. IADIZA, Mendoza, Argentina, pp 99-119.

RUBIO, M. C., D. SORIA, M. A. SALOMÓN, \& E. M. ABRAHAM. 2009. Delimitación de unidades geomorfológicas mediante la aplicación de técnicas de procesamiento digital de imágenes y SIG. Área no irrigada del departamento de Lavalle, Mendoza. Proyección 2: 1-33.

SCHWEINGRUBER, F. H., D. ECKSTEIN, F. SERRE-BACHET, \& O. U. BRÄKER. 1990. Identification, presentation and interpretation of event years and pointer years in dendrochronology. Dendrochronologia 8: 9-38.

TANENTZAP, A. J., E. P. MOUNTFORD, A. S. COOKE, \& D. A. COOMES. 2012. The more stems the merrier: Advantages of multi-stemmed architecture for the demography of understorey trees in a temperate broadleaf woodland. J. Ecol. 100: $171-183$.

VESK, P. A., \& M. WESTOBY. 2004. Funding the bud bank: a review of the costs of buds. Oikos 106: 200208.

WANG, T., H. REN, \& K. MA. 2005. Climatic signals in tree ring of Picea schrenkiana along an altitudinal gradient in the central Tianshan Mountains, northwestern China. Trees 19: 735-741.

WHITFORD, W. G. 2002. Ecology of desert systems. Academic Press.

WIGLEY, T. M. L., K. R. BRIFFA, \& P. D. JONES. 1984. On the average value of correlated time series, with applications in dendroclimatology and hydrometeorology. J. Clim. Appl. Meteorol. 23: 201-213.

YU, G., Y. LIU, X. WANG, \& K. MA, 2008. Agedependent tree-ring growth responses to climate in Qilian juniper (Sabina przewalskii Kom.). Trees 22: 197-204.

Recibido el 15 de septiembre de 2016, aceptado el 12 de enero de 2017. 
